\title{
A new SNR with TeV shell-type morphology: HESS J1731-347
}

HESS Collaboration, A. Abramowski ${ }^{1}$, F. Acero ${ }^{2}$, F. Aharonian ${ }^{3,4,5}$, A. G. Akhperjanian ${ }^{6,5}$, G. Anton 7 , A. Balzer ${ }^{7}$, A. Barnacka ${ }^{8,9}$, U. Barres de Almeida ${ }^{10, \star}$, Y. Becherini ${ }^{11,12}$, J. Becker $^{13}$, B. Behera ${ }^{14}, \mathrm{~K}$. Bernlöhr 3,15 , A. Bochow ${ }^{3}$, C. Boisson ${ }^{16}$, J. Bolmont ${ }^{17}$, P. Bordas ${ }^{18}$, J. Brucker ${ }^{7}$, F. Brun ${ }^{12}$, P. Brun ${ }^{9}$, T. Bulik ${ }^{19}$, I. Büsching ${ }^{20,13}$, S. Carrigan ${ }^{3}$, S. Casanova ${ }^{13}$, M. Cerruti ${ }^{16}$, P. M. Chadwick ${ }^{10}$, A. Charbonnier ${ }^{17}$, R. C. G. Chaves ${ }^{3}$, A. Cheesebrough ${ }^{10}$, L.-M. Chounet ${ }^{12}$, A. C. Clapson ${ }^{3}$, G. Coignet $^{21}$, G. Cologna ${ }^{14}$, J. Conrad ${ }^{22}$, M. Dalton ${ }^{15}$, M. K. Daniel ${ }^{10}$, I. D. Davids ${ }^{23}$, B. Degrange ${ }^{12}$, C. Deil ${ }^{3}$, H. J. Dickinson ${ }^{22}$, A. Djannati-Ataï ${ }^{11}$, W. Domainko ${ }^{3}$, L.O'C. Drury ${ }^{4}$, F. Dubois ${ }^{21}$, G. Dubus ${ }^{24}$, K. Dutson ${ }^{25}$, J. Dyks ${ }^{8}$, M. Dyrda ${ }^{26}$, K. Egberts ${ }^{27}$, P. Eger ${ }^{7}$, P. Espigat ${ }^{11}$, L. Fallon ${ }^{4}$, C. Farnier $^{2}$, S. Fegan ${ }^{12}$, F. Feinstein ${ }^{2}$, M. V. Fernandes ${ }^{1}$, A. Fiasson ${ }^{21}$, G. Fontaine ${ }^{12}$, A. Förster ${ }^{3}$, M. Füßling ${ }^{15}$, Y. A. Gallant ${ }^{2}$, H. Gast ${ }^{3}$, L. Gérard ${ }^{11}$, D. Gerbig ${ }^{13}$, B. Giebels ${ }^{12}$, J. F. Glicenstein ${ }^{9}$, B. Glück ${ }^{7}$, P. Goret ${ }^{9}$, D. Göring ${ }^{7}$, S. Häffner ${ }^{7}$, J. D. Hague ${ }^{3}$, D. Hampf ${ }^{1}$, M. Hauser ${ }^{14}$, S. Heinz ${ }^{7}$, G. Heinzelmann ${ }^{1}$, G. Henri' ${ }^{24}$, G. Hermann ${ }^{3}$, J. A. Hinton ${ }^{25}$, A. Hoffmann ${ }^{18}$, W. Hofmann ${ }^{3}$, P. Hofverberg ${ }^{3}$, M. Holler ${ }^{7}$, D. Horns ${ }^{1}$, A. Jacholkowska ${ }^{17}$, O. C. de Jager ${ }^{20}$, C. Jahn ${ }^{7}$, M. Jamrozy ${ }^{28}$, I. Jung ${ }^{7}$, M. A. Kastendieck ${ }^{1}$, K. Katarzyński ${ }^{29}$, U. Katz ${ }^{7}$, S. Kaufmann ${ }^{14}$, D. Keogh ${ }^{10}$, D. Khangulyan ${ }^{3}$, B. Khélifi ${ }^{12}$, D. Klochkov ${ }^{18}$, W. Kluźniak ${ }^{8}$, T. Kneiske ${ }^{1}$, Nu. Komin ${ }^{21}$, K. Kosack ${ }^{9}$, R. Kossakowski ${ }^{21}$, H. Laffon ${ }^{12}$, G. Lamanna ${ }^{21}$, D. Lennarz ${ }^{3}$, T. Lohse ${ }^{15}$, A. Lopatin ${ }^{7}$, C.-C. Lu ${ }^{3}$, V. Marandon ${ }^{11}$, A. Marcowith ${ }^{2}$, J. Masbou ${ }^{21}$, D. Maurin ${ }^{17}$, N. Maxted ${ }^{30}$, T. J. L. McComb ${ }^{10}$, M. C. Medina ${ }^{9}$, J. Méhault ${ }^{2}$,

R. Moderski ${ }^{8}$, E. Moulin ${ }^{9}$, C. L. Naumann ${ }^{17}$, M. Naumann-Godo ${ }^{9}$, M. de Naurois ${ }^{12}$, D. Nedbal ${ }^{31}$, D. Nekrassov ${ }^{3}$, N. Nguyen ${ }^{1}$, B. Nicholas ${ }^{30}$, J. Niemiec ${ }^{26}$, S. J. Nolan ${ }^{10}$, S. Ohm ${ }^{32,25,3}$, E. de Oña Wilhelmi ${ }^{3}$, B. Opitz ${ }^{1}$, M. Ostrowski ${ }^{28}$, I. Oya ${ }^{15}$, M. Panter ${ }^{3}$, M. Paz Arribas ${ }^{15}$, G. Pedaletti ${ }^{14}$, G. Pelletier ${ }^{24}$, P.-O. Petruccii ${ }^{24}$, S. Pita ${ }^{11}$, G. Pühlhofer ${ }^{18}$, M. Punch ${ }^{11}$, A. Quirrenbach ${ }^{14}$, M. Raue ${ }^{1}$, S. M. Rayner ${ }^{10}$, A. Reimer ${ }^{27}$, O. Reimer $^{27}$, M. Renaud $^{2}$, R. de los Reyes ${ }^{3}$, F. Rieger ${ }^{3,33}$, J. Ripken ${ }^{22}$, L. Rob ${ }^{31}$, S. Rosier-Lees ${ }^{21}$, G. Rowell ${ }^{30}$, B. Rudak ${ }^{8}$, C. B. Rulten ${ }^{10}$, J. Ruppel ${ }^{13}$, F. Ryde $^{34}$, V. Sahakian ${ }^{6,5}$, A. Santangelo ${ }^{18}$, R. Schlickeiser ${ }^{13}$, F. M. Schöck ${ }^{7}$, A. Schulz ${ }^{7}$, U. Schwanke ${ }^{15}$, S. Schwarzburg ${ }^{18}$, S. Schwemmer ${ }^{14}$, M. Sikora ${ }^{8}$, J. L. Skilton ${ }^{32}$, H. Sol ${ }^{16}$, G. Spengler ${ }^{15}$, Ł. Stawarz ${ }^{28}$, R. Steenkamp ${ }^{23}$, C. Stegmann ${ }^{7}$, F. Stinzing ${ }^{7}$, K. Stycz ${ }^{7}$, I. Sushch ${ }^{15, \star \star}$, A. Szostek ${ }^{28}$, J.-P. Tavernet ${ }^{17}$, R. Terrier ${ }^{11}$, M. Tluczykont ${ }^{1}$, K. Valerius ${ }^{7}$, C. van Eldik ${ }^{3}$, G. Vasileiadis ${ }^{2}$, C. Venter ${ }^{20}$, J. P. Vialle ${ }^{21}$, A. Viana ${ }^{9}$, P. Vincent ${ }^{17}$, H. J. Völk ${ }^{3}$, F. Volpe ${ }^{3}$, S. Vorobiov ${ }^{2}$, M. Vorster ${ }^{20}$, S. J. Wagner ${ }^{14}$, M. Ward ${ }^{10}$, R. White ${ }^{25}$, A. Wierzcholska ${ }^{28}$, M. Zacharias ${ }^{13}$, A. Zajczyk ${ }^{8,2}$, A. A. Zdziarski ${ }^{8}$, A. Zech ${ }^{16}$, and H.-S. Zechlin ${ }^{1}$

(Affiliations can be found after the references)

Received 31 December 2010 / Accepted 6 May 2011

\section{ABSTRACT}

Aims. The recent discovery of the radio shell-type supernova remnant (SNR), G353.6-0.7, in spatial coincidence with the unidentified TeV source HESS J1731-347 has motivated further observations of the source with the High Energy Stereoscopic System (HESS) Cherenkov telescope array to test a possible association of the $\gamma$-ray emission with the SNR.

Methods. With a total of $59 \mathrm{~h}$ of observation, representing about four times the initial exposure available in the discovery paper of HESS J1731-347, the $\gamma$-ray morphology is investigated and compared with the radio morphology. An estimate of the distance is derived by comparing the interstellar absorption derived from X-rays and the one obtained from ${ }^{12} \mathrm{CO}$ and $\mathrm{HI}$ observations.

Results. The deeper $\gamma$-ray observation of the source has revealed a large shell-type structure with similar position and extension $\left(r \sim 0.25^{\circ}\right)$ as the radio SNR, thus confirming their association. By accounting for the HESS angular resolution and projection effects within a simple shell model, the radial profile is compatible with a thin, spatially unresolved, rim. Together with RX J1713.7-3946, RX J0852.0-4622 and SN 1006, HESS J1731-347 is now the fourth SNR with a significant shell morphology at TeV energies. The derived lower limit on the distance of the SNR of $3.2 \mathrm{kpc}$ is used together with radio and X-ray data to discuss the possible origin of the $\gamma$-ray emission, either via inverse Compton scattering of electrons or the decay of neutral pions resulting from proton-proton interaction.

Key words. astroparticle physics - ISM: supernova remnants - cosmic rays

\section{Introduction}

In the survey of the Galactic plane carried out by the HESS experiment, many sources emitting at $\mathrm{TeV}$ energies remain

* Supported by CAPES Foundation, Ministry of Education of Brazil.

$\star \star$ Supported by Erasmus Mundus, External Cooperation Window. unidentified to date (e.g. Aharonian et al. 2008). Most of the sources are extended beyond the point spread function (PSF) of the HESS experiment $\left(\sim 0.06^{\circ}\right.$ for the analysis presented in this paper). The largest number of conclusive identifications so far can be attributed to pulsar wind nebulae (PWNe) as presented in e.g. Gallant et al. (2008). Recently, a new radio SNR, catalogued as G353.6-0.7, was discovered by Tian et al. (2008) to be 
in spatial coincidence with HESS J1731-347, one of the unidentified sources presented in Aharonian et al. (2008). The diameter of the radio shell is nearly $0.5^{\circ}$ which allows, given the brightness of the source and the HESS angular resolution of $\sim 0.06^{\circ}$, for a morphological comparison of the $\gamma$-ray source with the shell observed in radio. Moreover, at least up to the current date, no radio pulsar or X-ray PWN candidate was found that might alternatively explain the $\mathrm{TeV}$ emission. This situation should be compared to other $\gamma$-ray sources like HESS J1813-178 or HESS J1640-465 (Aharonian et al. 2006), which are also in spatial coincidence with radio SNR shells. However, for these latter sources a morphological identification with the radio shells is not possible, and the emission can plausibly originate from a PWN seen in X-rays as discussed in Funk et al. (2007b) for HESS J1640-465 and in Funk et al. (2007a); Gotthelf \& Halpern (2009) for HESS J1813-178.

Observations of the north-eastern part of HESS J1731-347 with the X-ray satellites XMM-Newton, Chandra, and Suzaku have confirmed an X-ray counterpart found in archival ROSAT data (presented in Aharonian et al. 2008; Tian et al. 2008). An $\mathrm{X}$-ray shell partly matching the radio morphology was found and the spectral analysis has revealed that the X-ray emission is of synchrotron origin, indicating that the shock wave of the SNR has accelerated electrons up to $\mathrm{TeV}$ energies (Acero et al. 2009b; Tian et al. 2010). A compact (unresolved) X-ray source XMMU J173203.3-344518 (Halpern \& Gotthelf 2010) was observed towards the geometrical center of the remnant and has spectral properties reminiscent of central compact objects (CCOs) found in several other supernova shells (e.g. Pavlov et al. 2004). A search for pulsations using the EPIC PN cameras onboard XMMNewton shows only marginal evidence of a $1 \mathrm{~s}$ period (Halpern \& Gotthelf 2010).

Given the recent discovery of G353.6-0.7, little is known about its age and distance. Tian et al. (2008) suggested a distance of $3.2 \pm 0.8 \mathrm{kpc}$ assuming that the SNR is at the same distance as the HII region G353.42-0.37.

Additional HESS observations, carried out since the discovery paper of HESS J1731-347 (Aharonian et al. 2008), allow to investigate the compatibility of the $\mathrm{TeV}$ source with the radio shell SNR G353.6-0.7. The observations and the data analysis are described in Sect. 2 and the morphological and spectral results in Sect. 3. The multi-wavelength counterparts of HESS J1731-347 are described in Sect. 4 and a general discussion is presented in Sect. 5 .

\section{HESS observations and analysis methods}

HESS is an array of four identical imaging atmospheric Cherenkov telescopes (IACTs) located in the Khomas Highland of Namibia $1800 \mathrm{~m}$ above sea level (Bernlöhr et al. 2003). The survey of the Galactic plane by the HESS collaboration has led to the discovery of the $\gamma$-ray source HESS J1731-347, presented as an unidentified extended source in Aharonian et al. (2008). In this first data set, $14 \mathrm{~h}$ of observation time were available. Additional dedicated observations were carried out in July 2007 and in July and August 2009 with zenith angles ranging from $9^{\circ}$ to $42^{\circ}$, the mean angle being $16.5^{\circ}$. The total HESS observation time for this target is $59 \mathrm{~h}$ after data quality cuts.

The data set was analyzed using the Model analysis (de Naurois \& Rolland 2009) which exploits the full pixel information by comparing the recorded shower images with a precalculated shower model using log-likelihood minimization. In comparison with conventional analysis techniques, no cleaning or parametrization of the image shape is required and the full

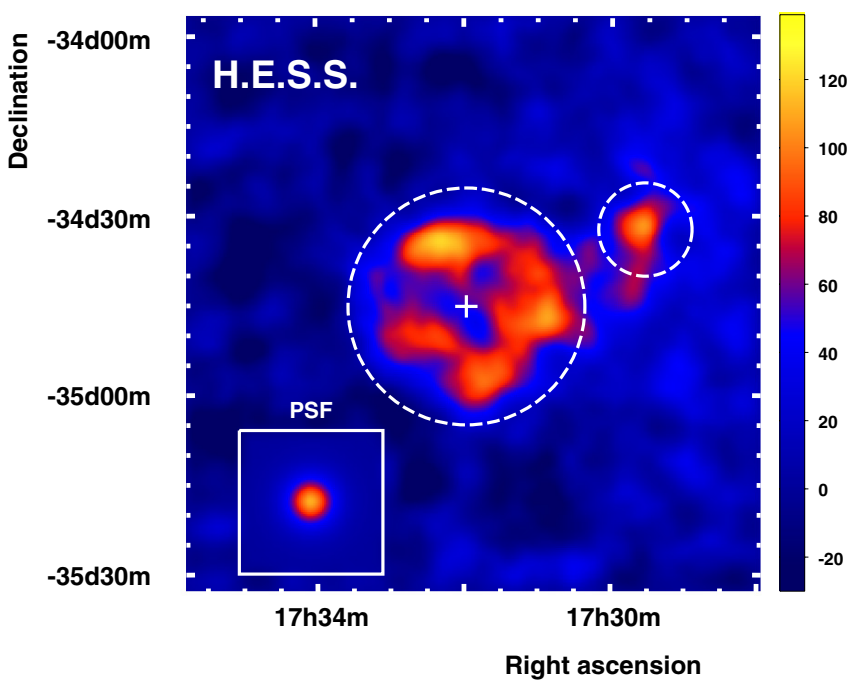

Fig. 1. TeV $\gamma$-ray excess map $\left(1.5^{\circ} \times 1.5^{\circ}\right)$ of the HESS J1731-347 region smoothed with a Gaussian width $\sigma=0.04^{\circ}$. The average HESS PSF for the dataset is shown in the inset. The regions used for the spectral analysis of HESS J1731-347 and HESS J1729-345 are respectively represented by the large and small dashed circles. The position of the central compact object detected in X-rays is shown with a white cross. The linear scale is in units of excess counts per smoothing Gaussian width. The transition between blue and red in the color scale is at the level of $4 \sigma$.

camera information is used. This method leads to a more accurate reconstruction and better background suppression than more conventional techniques and thus to an improved sensitivity.

Spectral and spatial analyses were carried out using a minimum image intensity of 60 photoelectrons (p.e.) resulting in an energy threshold of $240 \mathrm{GeV}$ and an angular resolution of $0.06^{\circ}$ (68\% containment radius). All results presented were crosschecked with a multivariate analysis (Ohm et al. 2009) using an independent calibration and gamma/hadron separation, which yielded consistent results. Unless otherwise quoted, the error bars in the following section are given at $1 \sigma$.

\section{TeV $\gamma$-rays analysis results}

The HESS excess map of the region of HESS J1731-347 is shown in Fig. 1 smoothed with a Gaussian of $\sigma=0.04^{\circ}$. For the background estimation in the image and in the morphology studies, the ring background method presented in Berge et al. (2007) was used. Because of the larger data set and the more sensitive reconstruction technique, the presented image is much more detailed than the one shown in the discovery paper (Aharonian et al. 2008). This reveals a complex region composed of a large and bright structure (HESS J1731-347), detected at 22 $\sigma$, with a suggestive shell-like morphology.

A smaller and fainter structure named HESS J1729-345 (detected at $8 \sigma$ ) is also observed, the properties of which are presented separately in Sect. 3.3.

\subsection{TeV energy morphology}

To further test the hypothesis of a shell morphology for HESS J1731-347 and its association with the radio SNR, radial and azimuthal profiles in radio and $\gamma$-rays were extracted centered 


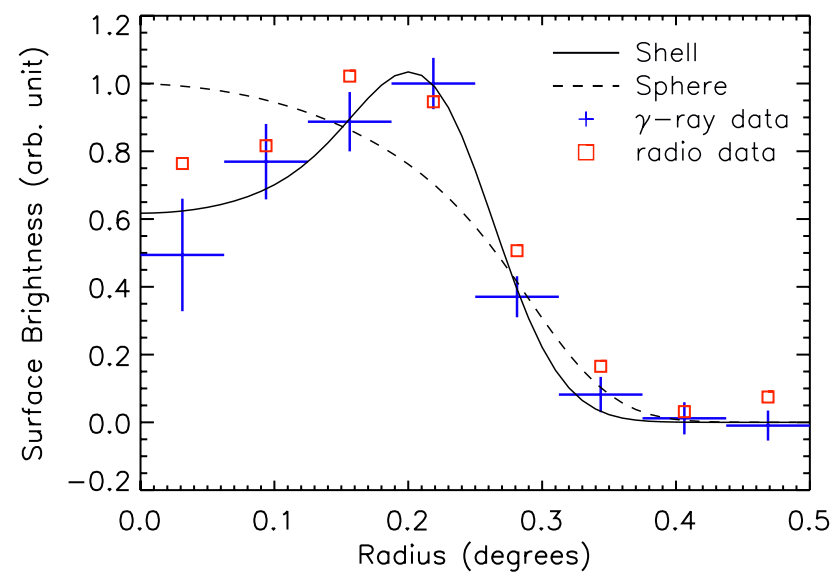

Fig. 2. The $\gamma$-ray excess and radio radial profiles are shown with blue crosses and red squares respectively. The best fits to the $\gamma$-ray data of a sphere and a shell model are overlaid. Both radial profiles are centered on the compact central object $\left(\alpha_{\mathrm{J} 2000}=17^{\mathrm{h}} 32 \mathrm{~m} 03^{\mathrm{s}}, \delta_{\mathrm{J} 2000}=\right.$ $\left.-34^{\circ} 45^{\prime} 18^{\prime \prime}\right)$.

on the position of the $\mathrm{CCO}\left(\alpha_{\mathrm{J} 2000}=17^{\mathrm{h}} 32^{\mathrm{m}} 03^{\mathrm{s}}, \delta_{\mathrm{J} 2000}=\right.$ $-34^{\circ} 45^{\prime} 18^{\prime \prime}$ ), also coincident with the geometrical center of the radio SNR. The profiles were derived from the uncorrelated $\gamma$ ray excess map and corrected for the field of view (FoV) acceptance.

For the $\gamma$-ray radial profile, the position angles ${ }^{1}$ from $270^{\circ}$ to $310^{\circ}$ were excluded, to avoid contamination from HESS J1729-345, and the resulting radial profile was compared with a sphere and a shell model. The first model is a uniformly emitting sphere of adjustable radius, projected on the sky and then folded with the PSF derived for this analysis $\left(r_{68 \%}=0.06^{\circ}\right)$. The shell model consists of a uniformly emitting shell of variable outer radius and thickness (defined as $r_{\text {outer }}-r_{\text {inner }}$ ) projected on the sky and then folded with the same PSF.

In the morphological test, the best fit statistically favors the shell model and the sphere model is ruled out at $3.9 \sigma\left(\chi^{2} /\right.$ d.o.f. $=$ $2.90 / 5$ and $28.12 / 6$ for the shell and sphere models respectively). In the case of a shell model, the best fit radius is $0.27^{\circ} \pm 0.02^{\circ}$ and the emission is compatible with a thin, spatially unresolved, shell with an upper limit thickness of $0.12^{\circ}$ (90\% confidence level).

To compare the $\mathrm{TeV}$ morphology with the shell seen in radio, the radio continuum map from the ATCA southern Galactic plane survey (SGPS) (Haverkorn et al. 2006) was smoothed to match the HESS spatial resolution and a radial profile was extracted (excluding point sources). The radio profile was then scaled by a normalization factor calculated as the ratio of the total number of excess $\gamma$-rays over the total radio flux on the whole remnant. The resulting profiles, presented in Fig. 2, show an extended emission in $\gamma$-rays similar to that seen in radio.

In contrast with RX J1713.7-3946 which is brighter in the North-West and SN 1006 that exhibits a bipolar morphology, the azimuthal profile of HESS J1731-347 (see Fig. 3) integrated for $r \leqslant 0.3^{\circ}$ shows no significant deviation from a flat profile $\left(\chi^{2} /\right.$ d.o.f. $\left.=8.8 / 9\right)$.

\footnotetext{
${ }^{1}$ Position angle $0^{\circ}$ corresponds to North and $90^{\circ}$ to East.
}

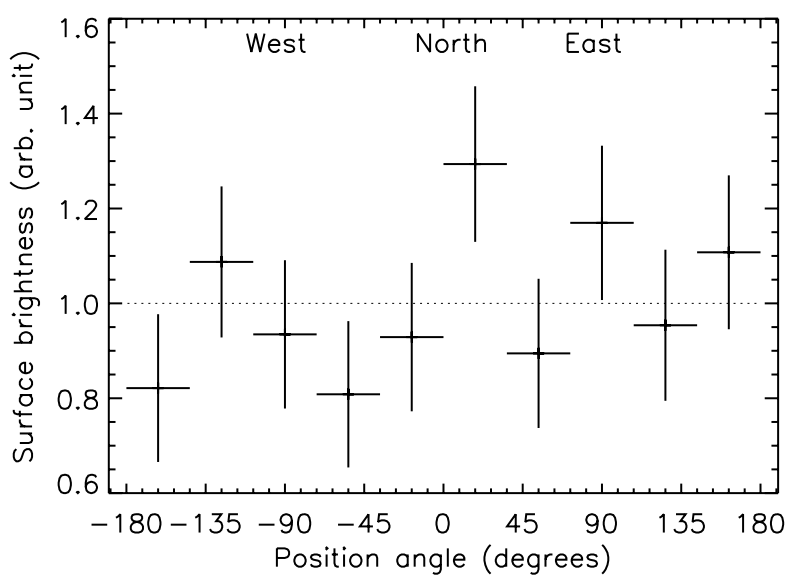

Fig. 3. Normalized azimuthal $\gamma$-ray excess profile restricted to radius $r \leq 0.3^{\circ}$ and using the same center as in Fig. 2. The brightness distribution is compatible with a flat profile.



Fig. 4. Differential energy spectra of HESS J1731-347 (filled circles) and HESS J1729-345 (open circles). The normalization for the second source has been divided by 10 for graphical purposes. Events were binned to reach a significance of at least $2 \sigma$. The best fit power-law models along with the residuals for HESS J1731-347 are also shown. The grey bands correspond to the range of the power-law fit, taking into account statistical errors.

\subsection{Spectral results}

The energy spectrum of the SNR was obtained by means of a forward-folding maximum likelihood fit (Piron et al. 2001) from a circular region centered on the $\mathrm{CCO}$, illustrated by the large dashed circle $\left(r=0.3^{\circ}\right)$ in Fig. 1, chosen to fully enclose the emission of the remnant. The background is estimated using the multiple reflected-regions technique where background events are selected from regions of the same size and shape as the source region and at equal angular distance from the observation position (Berge et al. 2007). The resulting spectrum, shown in Fig. 4, is well described by a power-law model (equivalent $\chi^{2} /$ d.o.f. $\left.=27.7 / 35\right)$ defined as $\mathrm{d} N / \mathrm{d} E=N_{0}\left(E / E_{0}\right)^{-\Gamma}$ where $E_{0}$ is the decorrelation energy (energy at which the correlation between the slope and the normalization vanishes). The best fit parameters, listed in Table 1 , result in an integrated $1-10 \mathrm{TeV}$ energy flux of $\left(6.91 \pm 0.75_{\text {stat }} \pm 1.38_{\text {syst }}\right) \times 10^{-12} \mathrm{erg} \mathrm{cm}^{-2} \mathrm{~s}^{-1}$. The flux measured here is lower than what has been derived 
Table 1. Best fit spectral parameters obtained for different extraction regions in HESS J1731-347.

\begin{tabular}{lcccc}
\hline \hline Region & Photon index $\Gamma$ & $\begin{array}{c}\text { Decorrelation energy } E_{0} \\
\text { TeV }\end{array}$ & $\begin{array}{c}\text { Normalization } N_{0} \\
10^{-12} \mathrm{~cm}^{-2} \mathrm{~s}^{-1} \mathrm{TeV}^{-1}\end{array}$ & $\begin{array}{c}1-10 \text { TeV integrated flux } \\
10^{-12} \mathrm{erg} \mathrm{cm}^{-2} \mathrm{~s}^{-1}\end{array}$ \\
\hline HESS J1731-347 & $2.32 \pm 0.06_{\text {stat }}$ & 0.783 & $4.67 \pm 0.19_{\text {stat }}$ & $6.91 \pm 0.75_{\text {stat }}$ \\
sub-region of HESS J1731-347 & $2.34 \pm 0.09_{\text {stat }}$ & 0.780 & $1.41 \pm 0.11_{\text {stat }}$ & $2.02 \pm 0.36_{\text {stat }}$ \\
HESS J1729-345 & $2.24 \pm 0.15_{\text {stat }}$ & 0.861 & $0.44 \pm 0.07_{\text {stat }}$ & $0.88 \pm 0.29_{\text {stat }}$ \\
\hline
\end{tabular}

Notes. The model used is a power-law of the form $\mathrm{d} N / \mathrm{d} E=N_{0}\left(E / E_{0}\right)^{-\Gamma}$. The systematic errors are conservatively estimated to be \pm 0.2 on the photon index and $20 \%$ on the flux. ${ }^{(a)}$ A spectral analysis corresponding to the FoV of the XMM-Newton data (see Fig. 5, center) has been carried out in order to build a SED.
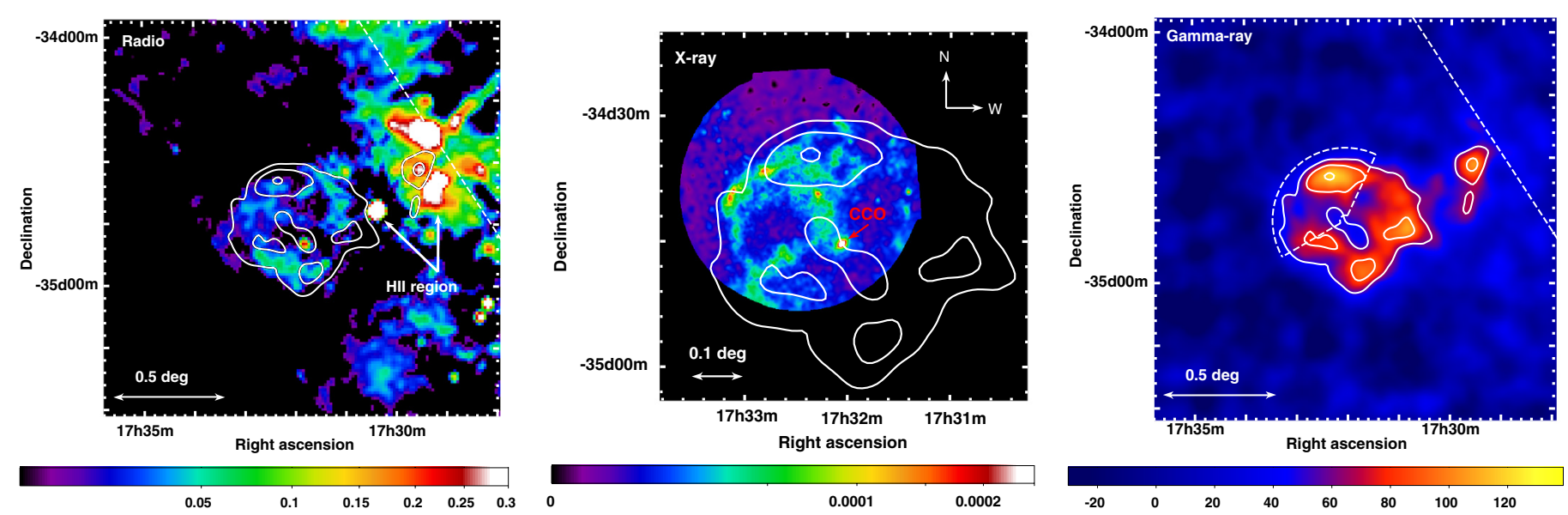

Fig. 5. Multi-wavelength view of the HESS J1731-347 region. The radio and the $\gamma$-ray image show the same field of view while the X-ray image is zoomed in order to show the details of the shell structure. The significance contours at 4,6 and $8 \sigma$ obtained with an integration radius of $0.06^{\circ}$ and the Galactic plane (white dashed line) are overlaid in the three panels. Left: ATCA radio map at $1.4 \mathrm{GHz}$ from the south Galactic plane survey (SGPS) in units of Jy/beam with a beam of 100". The HII regions G353.42-0.37 (left) and G353.381-0.114 (right) are marked with arrows. Middle: XMM-Newton observation of a sub-region of the SNR, in the $0.5-4.5 \mathrm{keV}$ energy band, using MOS instruments with units in $\mathrm{ph} / \mathrm{cm}^{2} / \mathrm{s} / \mathrm{arcmin}^{2}$. The position of the source XMMU J173203.3-344518, which is likely to be the CCO of the SNR is shown by the red arrow. Right: TeV $\gamma$-ray excess map of HESS J1731-347 smoothed with a Gaussian with $\sigma=0.04^{\circ}$. The region used to derive the radio flux and the spectral parameters in $\mathrm{X}$ - and $\gamma$-rays for the SED is also shown.

initially in Aharonian et al. (2008): $\left(16.2 \pm 3.6_{\text {stat }} \pm 3.2_{\text {syst }}\right) \times$ $10^{-12} \mathrm{erg} \mathrm{cm}^{-2} \mathrm{~s}^{-1}$ in the same energy band. However, the region of extraction in the discovery paper was much larger $\left(r=0.6^{\circ}\right.$ versus $r=0.3^{\circ}$ in this paper), including HESS J1729-345 and possibly some surrounding diffuse emission. A cross-check to derive the flux from the SNR only using the same data set as used in Aharonian et al. (2008) and following the original analysis method gave results consistent with the complete data set presented here thus confirming that the flux difference was mainly due to the choice of the integration region. A power-law model with an exponential cutoff was also tested which did not improve the quality of the fit (equivalent $\chi^{2} /$ d.o.f. $=24.0 / 34$ ).

\subsection{HESS J1729-345}

A $\gamma$-ray excess of $\mathrm{TeV}$ emission was found at the best fit position $\alpha_{\mathrm{J} 2000}=17^{\mathrm{h}} 29^{\mathrm{m}} 35^{\mathrm{s}}, \delta_{\mathrm{J} 2000}=-34^{\circ} 32^{\prime} 22^{\prime \prime}$ with a statistical error of $0.035^{\circ}$ and the source was therefore labeled HESS J1729-345.

The source is extended beyond the size of the PSF (Gaussian width $\sigma=0.12^{\circ} \pm 0.03^{\circ}$ ) and the region used to derive the spectral parameters is shown by the small dashed circle $\left(r=0.14^{\circ}\right)$ in Fig. 1. The spectrum obtained is well modeled by a powerlaw model (see Fig. 4) and the best fit parameters are listed in Table 1. The integrated flux in the $1-10 \mathrm{TeV}$ energy band is $\left(0.88 \pm 0.29_{\text {stat }} \pm 0.18_{\text {syst }}\right) \times 10^{-12} \mathrm{erg} \mathrm{cm}^{-2} \mathrm{~s}^{-1}$.

\section{Multi-wavelength counterparts}

One of the interesting characteristics of HESS J1731-347 is that non-thermal emission is clearly identified in radio, $\mathrm{X}$-rays and at $\mathrm{TeV}$ energies. In $\mathrm{X}$-rays however, the access to the spectral properties is limited to a subregion of the SNR as the coverage with the XMM-Newton, Chandra and Suzaku satellites is only partial, and the statistics in the ROSAT All Sky Survey data are too low. In order to study the spectral energy distribution (SED) of the source, the radio flux and the TeV spectral properties were extracted only from the region observed in X-rays (see region definition in Fig. 5, right). The multi-wavelength counterparts of HESS J1729-345 are discussed later in Sect. 4.5.

\subsection{Radio continuum}

The shell observed in radio is spatially coincident with the $\gamma$-ray shell and has a similar extent (see radial profile in Fig. 2). The flux obtained (excluding point sources) from the SGPS ATCA data in the region observed by the XMM-Newton pointing is $0.8 \pm 0.3 \mathrm{Jy}$ at $1420 \mathrm{MHz}$. The total radio flux for the SNR measured by Tian et al. (2008) is of $2.2 \pm 0.9 \mathrm{Jy}$. The compact HII region (G353.42-0.37) located to the West of the remnant at a distance of $3.2 \pm 0.8 \mathrm{kpc}$ (Tian et al. 2008) is indicated in Fig. 5 (left).

\subsection{X-rays}

In order to derive spectral information from the X-ray emission from the remnant, the XMM-Newton pointing obtained as a follow up of the HESS source (ObsId: 0405680201; PI: G. Pühlhofer) was analyzed. To clean the proton flare 
contamination during the observation, a histogram of the 10$12 \mathrm{keV}$ count rates of each camera was built. A Gaussian fit was then performed in order to remove time intervals where the count rates were beyond $3 \sigma$ from the mean value (Pratt $\&$ Arnaud 2002). The remaining exposure time after flare screening is $22 \mathrm{ks}$ out of the $25 \mathrm{ks}$ of observation for MOS and $15 \mathrm{ks}$ for PN. For the image generation, the instrumental background was derived from the compilation of blank sky observations by Carter $\&$ Read (2007) and renormalized in the 10-12 keV band over the whole FoV. The image resulting from the combination of the two MOS instruments is presented in Fig. 5 (middle). For this mosaic, the data from the PN instrument were not used because of straylight contamination to the North-East (photons singly reflected by the mirrors) from a bright X-ray source located outside the FoV. This results in some spurious arc features near the border of the FoV in the North-East.

The X-ray emission is characterized by extended emission which is concentrated in arc-like features, similar to broken shell seen from many shell-type SNRs. Some of the arcs partly coincide with the radio and $\gamma$-ray shell (see Fig. 5). Some of the structures could hint at an additional, smaller shell, but might also come from irregular SNR expansion in an inhomogeneous and/or dense medium (Blondin et al. 2001). A doubleshell structure is also observed in RX J1713.7-3946 in X-rays (Lazendic et al. 2004; Cassam-Chenaï et al. 2004; Acero et al. 2009a)

The spectral analysis of the diffuse X-ray emission was carried out using the Extended Source Analysis Software $\left(\mathrm{ESAS}^{2}\right)$ provided in the XMM-Newton Science Analysis System (SAS v9.0) to model the particle and instrumental backgrounds. The error bars in this section are quoted at $90 \%$ level confidence. For this analysis, the three instruments PN+MOS1+MOS2 were used and the regions were selected to avoid the straylight features.

The spectrum derived from the region covered by the FoV of $X M M-N e w t o n$ that is used for the SED is shown in Fig. 6. The emission is well represented by an absorbed power-law model and no emission lines were found (see also Tian et al. 2010). The best fit parameters obtained from a joint fit of MOS1, MOS2 and PN spectra are $N_{\mathrm{H}}=(1.08 \pm 0.02) \times 10^{22} \mathrm{~cm}^{-2}$, a spectral index $\Gamma=2.28 \pm 0.03$ and a normalization at $1 \mathrm{keV}$ $N_{0}=(1.37 \pm 0.05) \times 10^{-2} \mathrm{~cm}^{-2} \mathrm{~s}^{-1} \mathrm{keV}^{-1}$. A search for spatial spectral variations of the diffuse emission revealed that the power-law index is in most locations in the range $\Gamma=2.1-2.5$. Under the assumption of a pure power-law hypothesis, the absorption column significantly increases towards the Galactic plane from $N_{\mathrm{H}}=(0.93 \pm 0.05) \times 10^{22} \mathrm{~cm}^{-2}$ in the South-East region to $N_{\mathrm{H}}=(2.23 \pm 0.21) \times 10^{22} \mathrm{~cm}^{-2}$ in the North-West region (see Fig. 7; left). The errors on the absorption column shown in Fig. 7 (left) are ranging from $5 \%$ to $12 \%$.

The bright point source XMMU J173203.3-344518 lies at the geometrical center of the radio and $\gamma$-ray shell. Marginal evidence for a pulsation at a period of $1 \mathrm{~s}$ for the pulsar candidate and a faint nebula (radius of $30^{\prime \prime}$ ) whose spectral properties are compatible with a dust-scattered halo have been reported by Halpern \& Gotthelf (2010). As no optical or IR counterpart of the point source have been detected and as the Xray spectrum is well described by a blackbody emission model with $k T \sim 0.5 \mathrm{keV}$, the object is a good candidate to be the $\mathrm{CCO}$ of the SNR (Acero et al. 2009b; Halpern \& Gotthelf 2010; Tian et al. 2010).

\footnotetext{
2 http://xmm2.esac.esa.int/external/xmm_sw_cal/ background/epic_esas.shtml
}

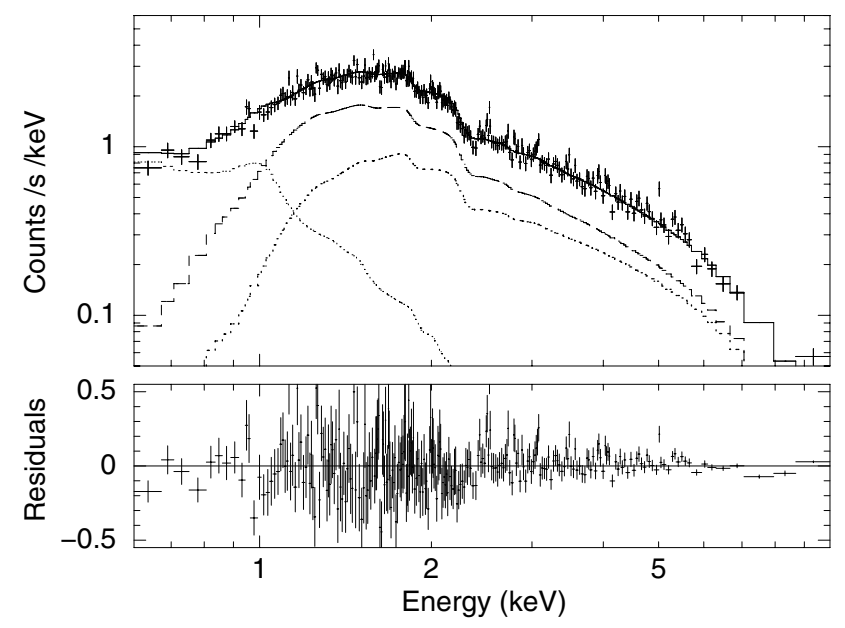

Fig. 6. X-ray spectrum, using the PN camera, extracted from the subregion of HESS J1731-347 shown in Fig. 5 (right). The non-thermal emission from the SNR is described by an absorbed power-law model (dashed line). The local astrophysical background, fitted to an off region outside the SNR, is modeled by two components (dotted lines). The low energy component is an APEC model (astrophysical plasma emission code, see http: //hea-www . harvard . edu/APEC) representing the background from the Local Bubble and the high-energy component is an absorbed power-law representing the hard X-ray background (unresolved AGNs, cataclysmic variables, etc.). The residuals of the total model (SNR+local astrophysical background) are shown in the lower panel and the $\chi^{2} / n$ d.o.f. is $1921 / 1569$.

\section{3. ${ }^{12} \mathrm{CO}(\mathrm{J}=1-0)$ and $\mathrm{HI}$}

The comparison of the absorption along the line of sight derived from X-ray data, ${ }^{12} \mathrm{CO}$ and $\mathrm{HI}$ observations can be used to constrain the distance to the SNR. The velocity spectra of the ${ }^{12} \mathrm{CO}$ emission (using data from the CfA survey, Dame et al. 2001) and the HI emission (using data from the SGPS survey, Haverkorn et al. 2006) derived from the region of highest X-ray absorption $\left(\alpha_{\mathrm{J} 2000}=17^{\mathrm{h}} 31^{\mathrm{m}} 43^{\mathrm{s}}, \delta_{\mathrm{J} 2000}=-34^{\circ} 34^{\prime} 58^{\prime \prime}\right)$ are shown in Fig. 8 (bottom).

In order to derive a lower limit on the integration distance required to match the $N_{\mathrm{H}}$ derived from X-rays, all the material is assumed to be at the near distance allowed by the Galactic rotation curve. Under this hypothesis, the cumulative absorption column derived from the atomic and molecular hydrogen shown in Fig. 8 (top) is similar to the one observed in X-rays, $N_{\mathrm{H}}=(2.23 \pm 0.21) \times 10^{22} \mathrm{~cm}^{-2}$, when integrating up to a radial velocity relative to the local standard of rest (LSR) of $-25 \mathrm{~km} \mathrm{~s}^{-1}$. The CO-to- $\mathrm{H}_{2}$ mass conversion factor and the HI brightness temperature to column density used are respectively of $1.8 \times 10^{20} \mathrm{~cm}^{-2} \mathrm{~K}^{-1} \mathrm{~km}^{-1} \mathrm{~s}$ (Dame et al. 2001) and $1.82 \times 10^{18} \mathrm{~cm}^{-2} \mathrm{~K}^{-1} \mathrm{~km}^{-1} \mathrm{~s}$ (Dickey \& Lockman 1990).

When integrating up to the same velocity, the map of $N_{\mathrm{H}}$ derived from the atomic and molecular hydrogen shown in Fig. 7 (right) exhibits an increase of absorption towards the Galactic plane similar to that in the X-ray absorption map in Fig. 7 (left). The peak of ${ }^{12} \mathrm{CO}$ emission at a LSR velocity of $-18 \mathrm{~km} \mathrm{~s}^{-1}$ is thus in the foreground of the SNR and is likely to be the cause of the gradient of absorption seen in X-rays. Using the circular Galactic rotation model of Hou et al. (2009) with a distance to the Galactic center of $8.0 \mathrm{kpc}$, the nearest distance corresponding to the LSR velocity of $-18 \mathrm{~km} \mathrm{~s}^{-1}$ is $3.2 \mathrm{kpc}$ thus setting a lower limit for the distance of the remnant. 

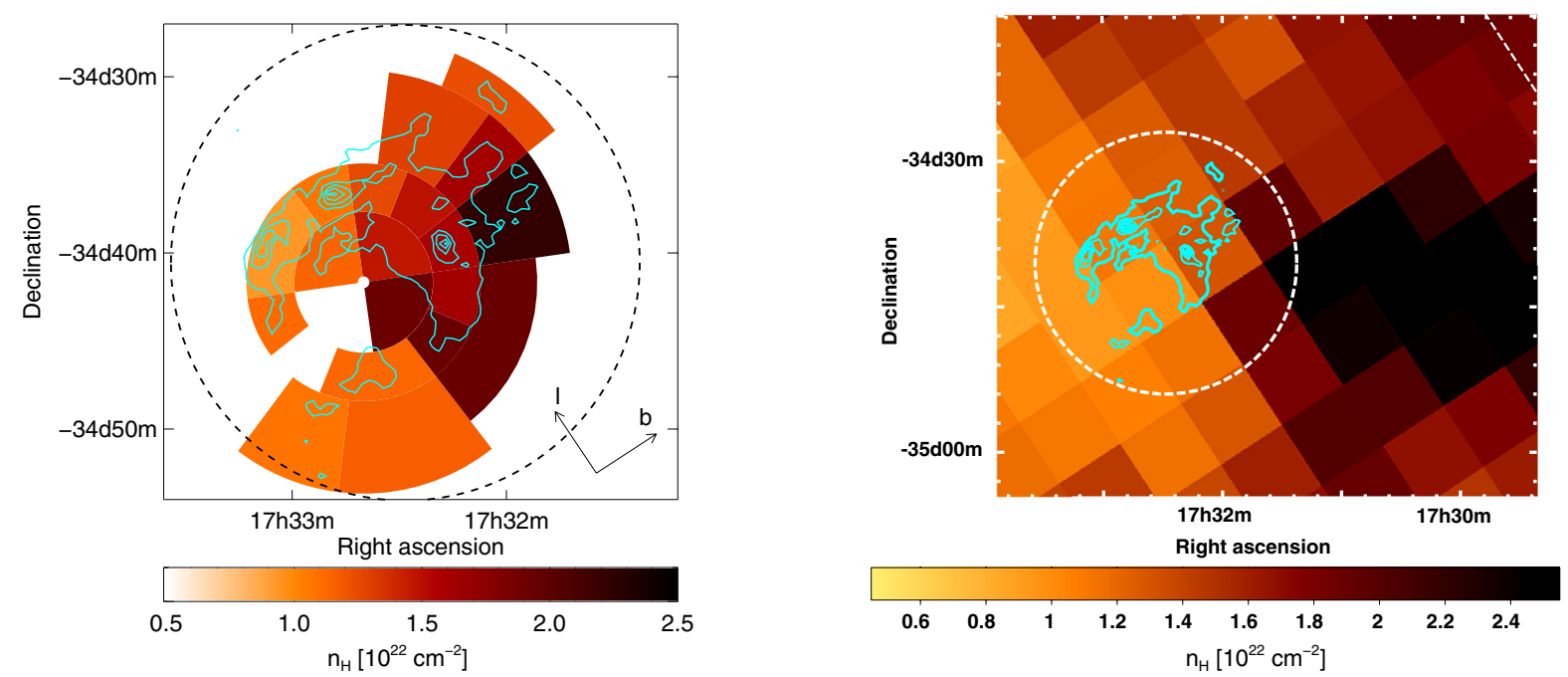

Fig. 7. Left: X-ray absorption map derived from a spectral fit to XMM-Newton data assuming an absorbed power-law model. A significant increase of $N_{\mathrm{H}}$ towards the Galactic plane is observed. Right: absorption column map derived from atomic and molecular hydrogen when integrating over radial velocities from $0 \mathrm{~km} \mathrm{~s}^{-1}$ to $-25 \mathrm{~km} \mathrm{~s}^{-1}$ (see Sect. 4.3 for more details). The Galactic plane is represented by the white dashed line. In both panels, the XMM-Newton field of view is represented by a dashed circle and the X-ray contours obtained from Fig. 5 (center) are overlaid.

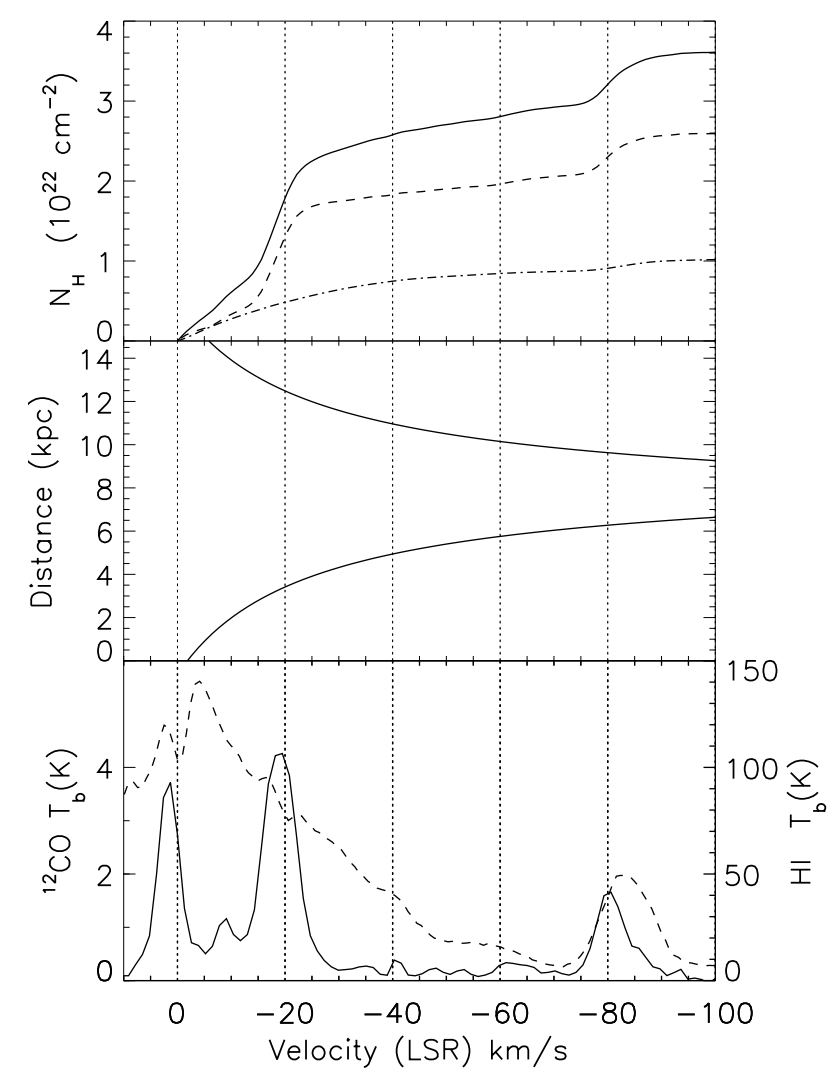

Fig. 8. Top: cumulative absorbing column density (solid line) as a function of radial velocity at the position of highest X-ray absorption (see Sect. 4.3). The relative contributions from the atomic and molecular hydrogen are represented by the dashed and dash-dotted lines respectively. Middle: rotation curve towards the same direction as derived from the model of Galactic rotation of Hou et al. (2009). Bottom: ${ }^{12} \mathrm{CO}$ (dashed line) and $\mathrm{HI}$ (dash-dotted line) spectra obtained the region highest X-ray absorption.

\subsection{GeV $\gamma$-rays}

In the Fermi-LAT first year catalog (Abdo et al. 2010) the source 1FGL J1729.1-3452c is found in the neighborhood of HESS J1731-347 as shown in Fig. 9. The Fermi source has an analysis flag that indicates that the source position moved beyond its $95 \%$ error ellipse when changing the model of diffuse emission. The ${ }^{12} \mathrm{CO}$ map in Fig. 9 shows that the Fermi source is located near a small scale gas clump that could be not well represented in the diffuse emission model. The position of the source presented in the catalog is to be used with caution and is therefore possibly not incompatible with HESS J1729-345.

The Fermi source has a photon spectral slope of $2.26 \pm 0.08$ and shows neither indication for spectral curvature nor time variability on a time scale of months (the catalog does not address shorter or longer time variations). This source is the closest Fermi detection near the newly discovered SNR and the flux derived in the Fermi catalog is used as an upper limit in the SED of the SNR in Fig. 10.

\subsection{Multi-wavelength counterparts for HESS J1729-345}

At radio wavelengths, the $\gamma$-ray contours of HESS J1729-345 lie near the HII region G353.381-0.114. Using HI radio recombination line data, the LSR velocity corresponding to this source is either $-54 \mathrm{~km} \mathrm{~s}^{-1}$ or $-82 \mathrm{~km} \mathrm{~s}^{-1}$ (Caswell \& Haynes 1987). In the latter case this HII region could be associated with the molecular cloud observed around velocities of $\sim-80 \mathrm{~km} \mathrm{~s}^{-1}$ (see Fig. 9). At X-ray energies, no archival dedicated observations were found, and no emission is detected in the ROSAT all sky survey, probably due to the high absorption in the line of sight. As discussed in the previous section, a Fermi source is found to lie close to HESS J1729-345.

\section{Discussion}

The newly discovered SNR HESS J1731-347 is in several ways comparable to RX J1713.7-3946 and RX J0852.0-4622. Those objects are X-ray synchrotron emitters and exhibit no thermal emission lines. A CCO is also found within those three SNRs indicating a core collapse SN. Moreover at a distance of $3.2 \mathrm{kpc}$ (see Sect. 4.3), the TeV luminosity of HESS J1731-347 in the $1-30 \mathrm{TeV}$ energy band is $1.07 \times(d / 3.2 \mathrm{kpc})^{2} 10^{34} \mathrm{erg} \mathrm{s}^{-1}$ which is similar to the luminosity of RX J1713.7-3946 (the brightest TeV shell SNR detected until now), of $0.81 \times 10^{34} \mathrm{erg} \mathrm{s}^{-1}$ 

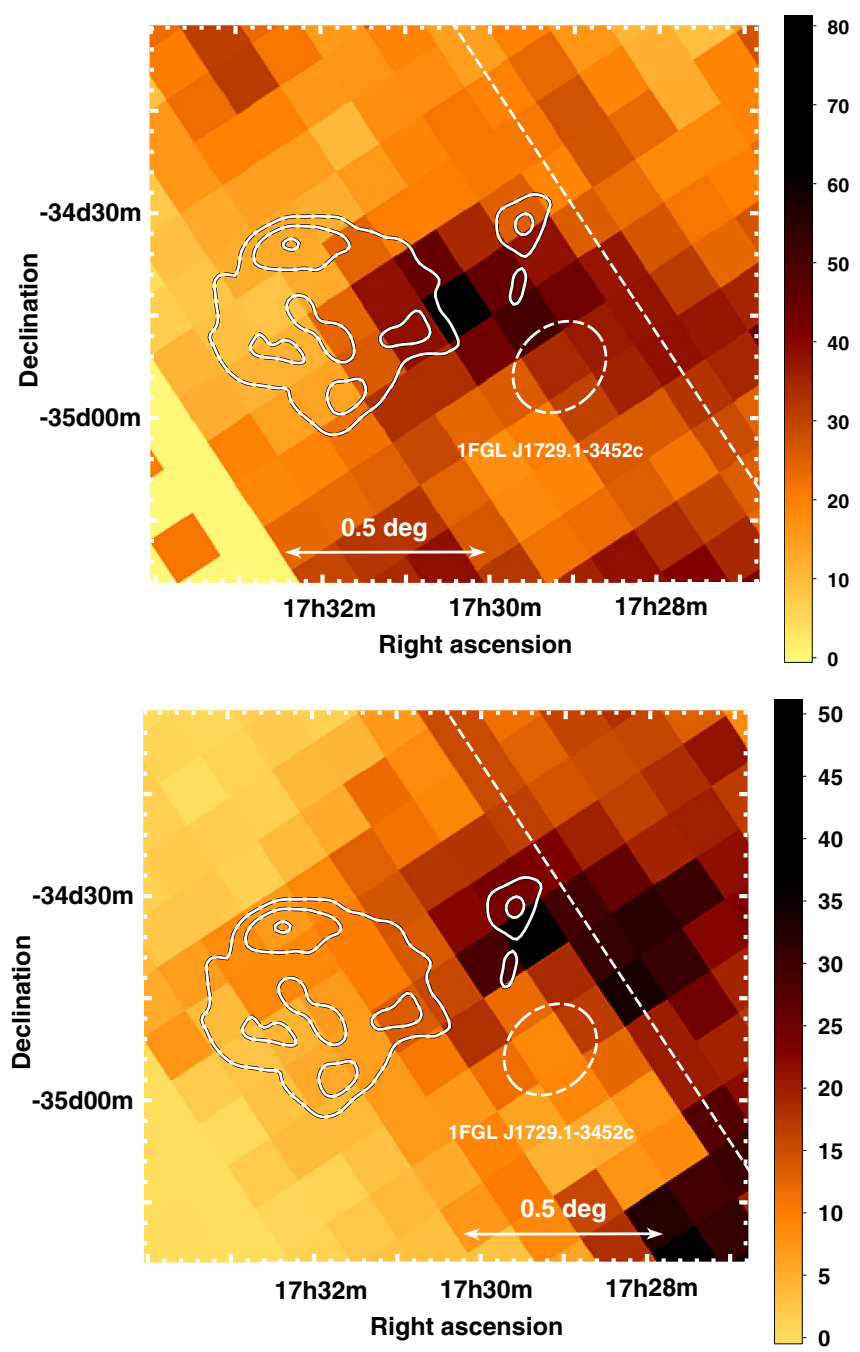

Fig. 9. ${ }^{12} \mathrm{CO}$ map of the vicinity of HESS J1731-347 integrated from LSR velocity $-13 \mathrm{~km} \mathrm{~s}^{-1}$ to $-25 \mathrm{~km} \mathrm{~s}^{-1}$ (top) and from $-75 \mathrm{~km} \mathrm{~s}^{-1}$ to $-87 \mathrm{~km} \mathrm{~s}^{-1}$ (bottom) respectively corresponding to the intervals of the second and third ${ }^{12} \mathrm{CO}$ peak shown in Fig. 8. The HESS significance contours from Fig. 5 together with the Galactic plane and the Fermi 95\% position confidence level contours presented in the 1 year catalog are overlaid. The linear scale is in units of $\mathrm{K} \mathrm{km} \mathrm{s}^{-1}$.

using a distance of $1 \mathrm{kpc}$ (Fukui et al. 2003; Cassam-Chenaï et al. 2004; Moriguchi et al. 2005) and slightly higher than that of RX J0852.0-4622 with $0.65 \times 10^{34} \mathrm{erg} \mathrm{s}^{-1}$ at a distance of $0.75 \mathrm{kpc}$ (Katsuda et al. 2008). A difference with RX J1713.7-3946 is that the flat $\gamma$-ray azimuthal profile of HESS J1731-347 (see Fig. 3) suggests that the remnant is evolving in a relatively uniform ambient medium and that it is not in interaction with the cloud (shown in Fig. 9, top) used to derive a lower limit to the distance of the SNR. This significantly differs from the case of RX J1713.7-3946 which exhibits much brighter $\gamma$-ray emission in the North-West where the shock is thought to interact with denser material.

The distance used for the luminosity is derived from the absorption in the foreground and provides only a lower limit of $3.2 \mathrm{kpc}$. However, as it is believed that supernova explosions are more likely to occur in the spiral arms of the Galaxy where the density of massive stars (i.e. SNR progenitors) is higher (Russeil 2003; Hou et al. 2009), it is likely that HESS J1731-347 could be located within the Scutum-Crux or Norma arms, which cross the line of sight at $l=353.5^{\circ}$ at $\sim 3.0$ and
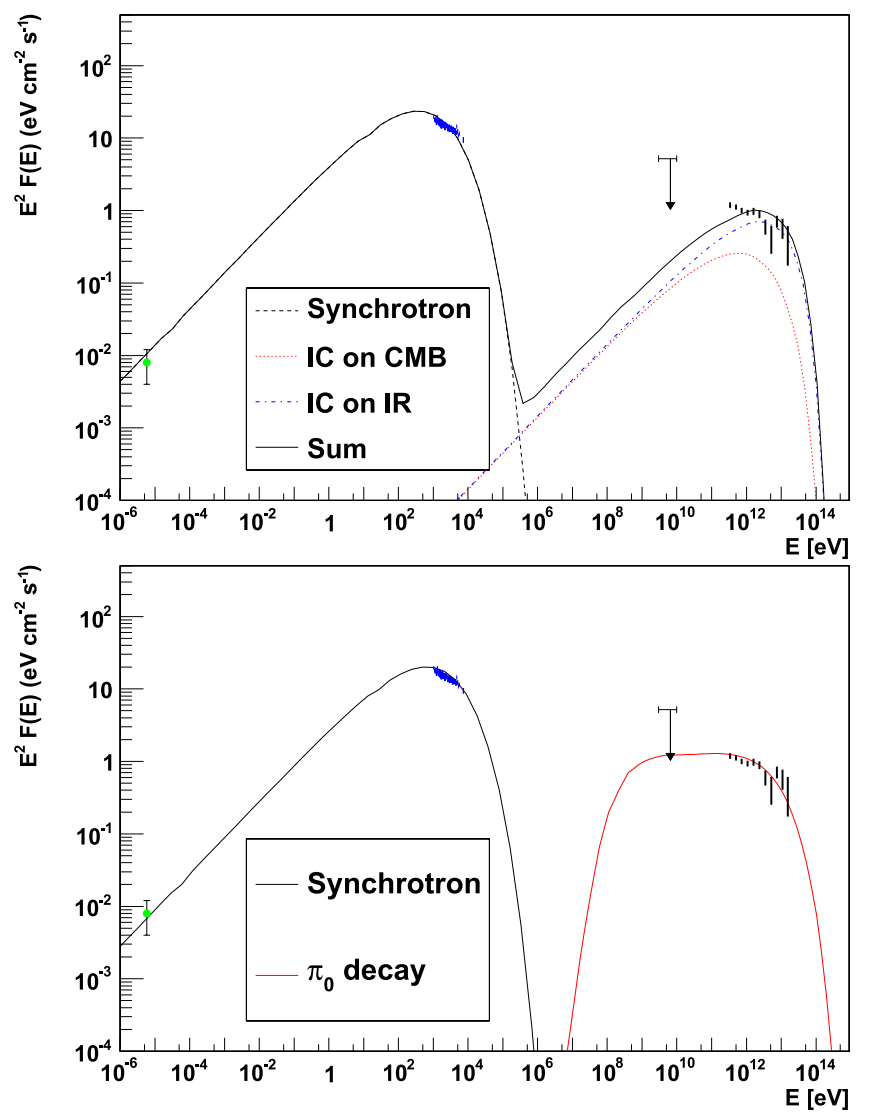

Fig. 10. Broadband SED for the sub-region of HESS J1731-347 that is observed in X-rays (see Fig. 5, right, for region definition). A purely leptonic (top) and a hadronic (bottom) scenario are shown and the corresponding parameters for both models are presented in Table 2. The infrared (IR) seed photons energy density and temperature were respectively set to $1 \mathrm{eV} \mathrm{cm}^{-3}$ and $40 \mathrm{~K}$ following the model from Porter et al. (2008) for a galactocentric radius of $4 \mathrm{kpc}$. The flux from the nearby Fermi source 1FGL J1729.1-3452c is represented and used as an upper limit.

$\sim 4.5 \mathrm{kpc}$ respectively (Hou et al. 2009). The next arm in the same line of sight is the Sagittarius arm lying at a distance of $12 \mathrm{kpc}$. This latter possibility for the location of the SNR would lead to a much higher $\gamma$-ray luminosity, an order of magnitude higher than RX J1713.7-3946. Also at such a distance, the physical size of the remnant would exceed $50 \mathrm{pc}$, substantially larger than other TeV shell SNRs whose physical size is $\lesssim 15$ pc. As a result it is reasonable to believe that the real distance to the SNR should not be much larger than the derived lower limit of $3.2 \mathrm{kpc}$.

The radio flux and the X-and $\gamma$-ray spectra derived in Sect. 4 from the sub-region of HESS J1731-347 that is covered by the FoV of XMM-Newton were combined in the SED presented in Fig. 10. The X-ray data were corrected for the interstellar absorption with $N_{\mathrm{H}}=1.08 \times 10^{22} \mathrm{~cm}^{-2}$. To model the SED, a simple one-zone stationary model (presented in Acero et al. 2010) was used. In this model, the spectrum of electrons and protons is represented by a power-law of slope $s$ with exponential cutoffs at energies $E_{\mathrm{c}, \mathrm{e}}$ and $E_{\mathrm{c}, \mathrm{p}}$ for the electrons and protons respectively. For the modeling of the object, it is assumed that the measured multi-wavelength emission from the sub-region of HESS J1731-347 is entirely coming from the SNR located at a distance of $3.2 \mathrm{kpc}$. As this distance is only a lower limit, the total energy of accelerated particles $\left(W_{\mathrm{e}}\right.$ and $\left.W_{\mathrm{p}}\right)$ in the SNR should also be viewed as lower limits. 
Table 2. List of the parameters used for the spectral energy distribution modeling presented in Fig. 10.

\begin{tabular}{lccccc}
\hline \hline Model & $\begin{array}{c}E_{\mathrm{c}, \mathrm{e}} \\
\mathrm{TeV}\end{array}$ & $\begin{array}{c}E_{\mathrm{c}, \mathrm{p}} \\
\mathrm{TeV}\end{array}$ & $\begin{array}{c}W_{\mathrm{e}} \\
10^{47} \mathrm{erg}\end{array}$ & $\begin{array}{c}W_{\mathrm{p}} \\
10^{50} \mathrm{erg}\end{array}$ & $\begin{array}{c}B \\
\mu \mathrm{G}\end{array}$ \\
\hline leptonic & 18 & - & 1.1 & - & 25 \\
hadronic & 16 & 100 & 0.25 & 2.0 & 50 \\
\hline
\end{tabular}

Notes. The spectral slope are fixed at 2.0 for the electron and the proton distribution. The density of the ambient medium was set to $1 \mathrm{~cm}^{-3}$ in the case of the hadronic model.

In the pure leptonic scenario, the slope of the electrons is constrained by the radio and the X-ray synchrotron emission between 1.9 and 2.1 and the strength of the magnetic field required to reproduce the ratio of observed synchrotron and IC emission lies between 20 and $30 \mu \mathrm{G}$ for $15 \leq E_{\mathrm{c}, \mathrm{e}} \leq 25 \mathrm{TeV}$. Although the relative ratio of radio, $\mathrm{X}$ - and $\mathrm{TeV}$ fluxes can be fairly well reproduced by this leptonic scenario, the model is inadequate to account for the X-ray and the $\gamma$-ray spectral slope as illustrated in Fig. 10 (top). The corresponding parameters for the latter model are summarized in Table 2.

This limitation no longer occurs in a scenario where the $\mathrm{TeV}$ emission is dominated by hadronic processes as the X-and $\gamma$-ray emission are now independent and both spectral slopes can be reproduced as shown in Fig. 10 (bottom). Moreover, the strength of the magnetic field can be increased as it is no longer fixed by the $\mathrm{X} / \gamma$ ratio. In order to reproduce the observed TeV flux, the total energy in high-energy protons $(E \geq 1 \mathrm{GeV})$ assuming a spectral slope of 2.0 is $W_{\mathrm{p}}=2 \times 10^{50}\left(\mathrm{n} / 1 \mathrm{~cm}^{-3}\right)^{-1}(d / 3.2 \mathrm{kpc})^{2} \mathrm{erg}$. It should be noted that this energy content only represents a subregion of the SNR accounting for $\sim 1 / 3$ of the total $\mathrm{TeV}$ flux (see Table 1) implying that the total energy transferred to accelerated protons in the whole SNR is a substantial fraction of the energy available in the remnant for $n \sim 1 \mathrm{~cm}^{-3}$. For this energetic reason, gas densities much below this value appear incompatible with the hadronic emission scenario.

Although it is not possible to measure the density of the ambient medium surrounding the SNR as no X-ray thermal emission is detected, an upper limit on the density can be derived. In order to do so, a thermal component, whose normalization is fixed for a given density using the method presented in Acero et al. (2007, Sect. 3.1), is added to the X-ray spectrum. The shocked ambient medium is assumed to be in a non equilibrium ionization state with an ionization timescale parameter $\tau=10^{9} \mathrm{~cm}^{-3} \mathrm{~s}$ and an electron plasma temperature $k T_{\mathrm{e}}=1 \mathrm{keV}$. Such values are commonly observed in other young SNRs for which the X-ray emission of the shocked ambient medium has been studied as in e.g. RCW86 (see Vink et al. 2006). For the given parameters, the derived upper limit ( $90 \%$ confidence level) on the ambient medium density is $10^{-2} \mathrm{~cm}^{-3}$. In the case of a lower temperature $\left(k T_{\mathrm{e}}=0.15 \mathrm{keV}\right)$, an upper limit of $1 \mathrm{~cm}^{-3}$ is reached.

For a density of $1 \mathrm{~cm}^{-3}$ the corresponding shock speed and age of the SNR would be $\sim 410 \mathrm{~km} \mathrm{~s}^{-1}$ and $14000 \mathrm{yrs}$ in order to match a physical radius of $R_{\text {shock }}=15 \mathrm{pc}\left(0.27^{\circ}\right.$ at $\left.3.2 \mathrm{kpc}\right)$, for a $\mathrm{SN}$ explosion of $E_{\mathrm{SN}}=1 \times 10^{51} \mathrm{erg}$ with a mass of ejecta of $5 M_{\odot}$ using equations from Truelove \& McKee (1999). However, this shock speed is an order of magnitude lower than what has been measured in other bright synchrotron emitting SNRs like $\mathrm{SN} 1006\left(V_{\mathrm{sh}}=5000 \pm 400 \mathrm{~km} \mathrm{~s}^{-1}\right.$ at a distance of $2.2 \mathrm{kpc}$; Katsuda et al. 2009), RCW $86\left(V_{\mathrm{sh}}=6000 \pm 3000 \mathrm{~km} \mathrm{~s}^{-1}\right.$; Helder et al. 2009), CasA $\left(V_{\mathrm{sh}}=4900 \mathrm{~km} \mathrm{~s}^{-1}\right.$; Patnaude \& Fesen 2009) or Tycho $\left(V_{\mathrm{sh}}=3000 \pm 1000 \mathrm{~km} \mathrm{~s}^{-1}\right.$ at a distance of $2.3 \mathrm{kpc}$; Katsuda et al. 2010). As a rough estimate, the required density to reproduce a canonical shock speed of
$3000 \mathrm{~km} \mathrm{~s}^{-1}$ using the aforementioned SN parameters is of the order of $0.01 \mathrm{~cm}^{-3}$ (compatible with the upper limit derived from the lack of thermal X-ray emission in the previous paragraph) for a corresponding age of $\sim 2500$ yrs.

To summarize, the presented static one-zone model suffers from limitations in both scenarios. In the leptonic case, the model allows to estimate the average B-field $(\sim 25 \mu \mathrm{G})$ and the total energy in accelerated electrons present in the shell of the SNR but fails to reproduce the observed X-ray and $\gamma$-ray spectral slope. In the hadronic model, the high medium density required to reproduce the observed $\mathrm{TeV}$ flux is hardly compatible with the hydrodynamics of the SNR. More detailed models using nonlinear diffuse shock acceleration theory have been developed (e.g. Zirakashvili \& Aharonian 2010; Ellison et al. 2010) and would provide more accurate predictions than the simple model presented here. It should be noted that the considered model does not take into account evolution related to radiative cooling which could yield a steeper gamma-ray spectrum, in better agreement with the data. Also, the presented scenarios do not cover possible non-homogeneous surroundings such as wind bubble blown by the progenitor. Such detailed spectral and evolutionary modeling depends on many poorly known parameters and is therefore beyond the scope of the present discussion.

Concerning the source HESS J1729-345, detected in the vicinity of the SNR, the presented multi-wavelength data do not provide a clear understanding of the nature of the object. The closest structures located near the $\gamma$-ray emission are the HII region G353.381-0.114 (seen in the radio in Fig. 5, left) and a molecular gas clump observed in ${ }^{12} \mathrm{CO}$ (see Fig. 9, bottom) when integrating around a LSR velocity of $-80 \mathrm{~km} \mathrm{~s}^{-1}$ (corresponding to near and far kinematic distances of $\sim 6$ and $\sim 10 \mathrm{kpc}$ respectively). If the $\gamma$-ray source HESS J1729-345 is associated with those gas structures, it would therefore not be associated with the SNR HESS J1731-347 thought to lie at a closer distance.

\section{Conclusion}

The newly discovered SNR HESS J1731-347 exhibits a significant shell morphology spatially resolved by HESS, similar to the one observed in radio. Together with RX J1713.7-3946, RX J0852.0-4622 and SN 1006, HESS J1731-347 is now the fourth $^{3} \mathrm{TeV} \gamma$-ray source to join this small but growing class. A lower limit to the distance of the SNR of $3.2 \mathrm{kpc}$ was obtained by comparing the absorption derived from the X-rays and from $\mathrm{HI}$ and ${ }^{12} \mathrm{CO}$ observations.

The multi-wavelength emission from the SNR, detected in radio, $\mathrm{X}$-rays and $\gamma$-rays, was combined in an SED to investigate the origin of the $\gamma$-ray emission assuming that the broadband emission stems from the same region (one-zone model). While the measured fluxes can be accounted for in a purely leptonic model with a magnetic field of the order of $25 \mu \mathrm{G}$, this simple model fails to reproduce the spectral shape of the X-and $\gamma$-ray emission. A second model that assumes that the $\mathrm{TeV}$ emission is produced by hadronic processes is able to reproduce the spectral slopes in $\mathrm{X}$ - and $\gamma$-rays at the cost of requiring that a large fraction of the kinetic energy of the explosion must be transferred to the accelerated protons and a high ambient medium density of $n \sim 1 \mathrm{~cm}^{-3}$ for $d \geq 3.2 \mathrm{kpc}$. Moreover for such a density, the corresponding shock speed of the SNR would be an order of magnitude lower than in other SNRs exhibiting bright synchrotron emission.

\footnotetext{
3 With the current statistics, the shell morphology of RCW86 is not statistically significant (Aharonian et al. 2009).
} 
Acknowledgements. The support of the Namibian authorities and of the University of Namibia in facilitating the construction and operation of HESS is gratefully acknowledged, as is the support by the German Ministry for Education and Research (BMBF), the Max Planck Society, the French Ministry for Research, the CNRS-IN2P3 and the Astroparticle Interdisciplinary Programme of the CNRS, the UK Science and Technology Facilities Council (STFC), the IPNP of the Charles University, the Polish Ministry of Science and Higher Education, the South African Department of Science and Technology and National Research Foundation, and by the University of Namibia. We appreciate the excellent work of the technical support staff in Berlin, Durham, Hamburg, Heidelberg, Palaiseau, Paris, Saclay, and in Namibia in the construction and operation of the equipment. Article based on observations obtained with $X M M$ Newton, an ESA science mission with instruments and contributions directly funded by ESA Member States and NASA.

\section{References}

Abdo, A. A., Ackermann, M., Ajello, M., et al. 2010, ApJS, 188, 405 Acero, F., Ballet, J., \& Decourchelle, A. 2007, A\&A, 475, 883 Acero, F., Ballet, J., Decourchelle, A., et al. 2009a, A\&A, 505, 157 Acero, F., Pühlhofer, G., Klochkov, D., et al. 2009b [arXiv: 0907.0642] Acero, F., Aharonian, F., Akhperjanian, A. G., et al. 2010, A\&A, 516, A62 Aharonian, F., Akhperjanian, A. G., Bazer-Bachi, A. R., et al. 2006, ApJ, 636, 777

Aharonian, F., Akhperjanian, A. G., Barres de Almeida, U., et al. 2008, A\&A, 477,353

Aharonian, F., Akhperjanian, A. G., de Almeida, U. B., et al. 2009, ApJ, 692, 1500

Berge, D., Funk, S., \& Hinton, J. 2007, A\&A, 466, 1219

Bernlöhr, K., Carrol, O., Cornils, R., et al. 2003, Astrop. Phys., 20, 111

Blondin, J. M., Chevalier, R. A., \& Frierson, D. M. 2001, ApJ, 563, 806

Carter, J. A., \& Read, A. M. 2007, A\&A, 464, 1155

Cassam-Chenaï, G., Decourchelle, A., Ballet, J., et al. 2004, A\&A, 427, 199

Caswell, J. L., \& Haynes, R. F. 1987, A\&A, 171, 261

Dame, T. M., Hartmann, D., \& Thaddeus, P. 2001, ApJ, 547, 792

de Naurois, M., \& Rolland, L. 2009, Astrop. Phys., 32, 231

Dickey, J. M., \& Lockman, F. J. 1990, ARA\&A, 28, 215

Ellison, D. C., Patnaude, D. J., Slane, P., \& Raymond, J. 2010, ApJ, 712, 287

Fukui, Y., Moriguchi, Y., Tamura, K., et al. 2003, PASJ, 55, L61

Funk, S., Hinton, J. A., Moriguchi, Y., et al. 2007a, A\&A, 470, 249

Funk, S., Hinton, J. A., Pühlhofer, G., et al. 2007b, ApJ, 662, 517

Gallant, Y. A., Carrigan, S., Djannati-Ataï, A., et al. 2008, in 40 Years of

Pulsars: Millisecond Pulsars, Magnetars and More, ed. C. Bassa, Z. Wang,

A. Cumming, \& V. M. Kaspi, Am. Inst. Phys. Conf. Ser., 983, 195

Gotthelf, E. V., \& Halpern, J. P. 2009, ApJ, 700, L158

Halpern, J. P., \& Gotthelf, E. V. 2010, ApJ, 710, 941

Haverkorn, M., Gaensler, B. M., McClure-Griffiths, N. M., Dickey, J. M., \& Green, A. J. 2006, ApJS, 167, 230

Helder, E. A., Vink, J., Bassa, C. G., et al. 2009, Science, 325, 719

Hou, L. G., Han, J. L., \& Shi, W. B. 2009, A\&A, 499, 473

Katsuda, S., Tsunemi, H., \& Mori, K. 2008, ApJ, 678, L35

Katsuda, S., Petre, R., Long, K. S., et al. 2009, ApJ, 692, L105

Katsuda, S., Petre, R., Hughes, J. P., et al. 2010, ApJ, 709, 1387

Lazendic, J. S., Slane, P. O., Gaensler, B. M., et al. 2004, ApJ, 602, 271

Moriguchi, Y., Tamura, K., Tawara, Y., et al. 2005, ApJ, 631, 947

Ohm, S., van Eldik, C., \& Egberts, K. 2009, Astrop. Phys., 31, 383

Patnaude, D. J., \& Fesen, R. A. 2009, ApJ, 697, 535

Pavlov, G. G., Sanwal, D., \& Teter, M. A. 2004, in Young Neutron Stars and

Their Environments, ed. F. Camilo, \& B. M. Gaensler, IAU Symp., 218, 239

Piron, F., Djannati-Atai, A., Punch, M., et al. 2001, A\&A, 374, 895

Porter, T. A., Moskalenko, I. V., Strong, A. W., Orlando, E., \& Bouchet, L. 2008, ApJ, 682, 400

Pratt, G. W., \& Arnaud, M. 2002, A\&A, 394, 375

Russeil, D. 2003, A\&A, 397, 133

Tian, W. W., Leahy, D. A., Haverkorn, M., \& Jiang, B. 2008, ApJ, 679, L85

Tian, W. W., Li, Z., Leahy, D. A., et al. 2010, ApJ, 712, 790

Truelove, J. K., \& McKee, C. F. 1999, ApJS, 120, 299

Vink, J., Bleeker, J., van der Heyden, K., et al. 2006, ApJ, 648, L33

Zirakashvili, V. N., \& Aharonian, F. A. 2010, ApJ, 708, 965

1 Universität Hamburg, Institut für Experimentalphysik, Luruper Chaussee 149, 22761 Hamburg, Germany

${ }^{2}$ Laboratoire Univers et Particules de Montpellier, Université Montpellier 2, CNRS/IN2P3, CC 72, Place Eugène Bataillon, 34095 Montpellier Cedex 5, France

e-mail: facero@in2p3.fr
3 Max-Planck-Institut für Kernphysik, PO Box 103980, 69029 Heidelberg, Germany

${ }^{4}$ Dublin Institute for Advanced Studies, 31 Fitzwilliam Place, Dublin 2, Ireland

5 National Academy of Sciences of the Republic of Armenia, Yerevan, Armenia

6 Yerevan Physics Institute, 2 Alikhanian Brothers St., 375036 Yerevan, Armenia

7 Universität Erlangen-Nürnberg, Physikalisches Institut, ErwinRommel-Str. 1, 91058 Erlangen, Germany

8 Nicolaus Copernicus Astronomical Center, ul. Bartycka 18, 00-716 Warsaw, Poland

9 CEA Saclay, DSM/IRFU, 91191 Gif-Sur-Yvette Cedex, France

10 University of Durham, Department of Physics, South Road, Durham DH1 3LE, UK

11 Astroparticule et Cosmologie (APC), CNRS, Université Paris 7 Denis Diderot, 10 rue Alice Domon et Léonie Duquet, 75205 Paris Cedex 13, France (UMR 7164: CNRS, Université Paris VII, CEA, Observatoire de Paris)

12 Laboratoire Leprince-Ringuet, École Polytechnique, CNRS/IN2P3, 91128 Palaiseau, France

13 Institut für Theoretische Physik, Lehrstuhl IV: Weltraum und Astrophysik, Ruhr-Universität Bochum, 44780 Bochum, Germany

14 Landessternwarte, Universität Heidelberg, Königstuhl, 69117 Heidelberg, Germany

15 Institut für Physik, Humboldt-Universität zu Berlin, Newtonstr. 15, 12489 Berlin, Germany

16 LUTH, Observatoire de Paris, CNRS, Université Paris Diderot, 5 Place Jules Janssen, 92190 Meudon, France

17 LPNHE, Université Pierre et Marie Curie Paris 6, Université Denis Diderot Paris 7, CNRS/IN2P3, 4 Place Jussieu, 75252 Paris Cedex 5, France

18 Institut für Astronomie und Astrophysik, Universität Tübingen, Sand 1, 72076 Tübingen, Germany

19 Astronomical Observatory, The University of Warsaw, Al. Ujazdowskie 4, 00-478 Warsaw, Poland

${ }^{20}$ Unit for Space Physics, North-West University, Potchefstroom 2520, South Africa

${ }^{21}$ Laboratoire d'Annecy-le-Vieux de Physique des Particules, Université de Savoie, CNRS/IN2P3, 74941 Annecy-le-Vieux, France

22 Oskar Klein Centre, Department of Physics, Stockholm University, Albanova University Center, 10691 Stockholm, Sweden

23 University of Namibia, Department of Physics, Private Bag 13301, Windhoek, Namibia

${ }^{24}$ Laboratoire d'Astrophysique de Grenoble, INSU/CNRS, Université Joseph Fourier, BP 53, 38041 Grenoble Cedex 9, France

25 Department of Physics and Astronomy, The University of Leicester, University Road, Leicester, LE1 7RH, UK

${ }^{26}$ Instytut Fizyki Jạdrowej PAN, ul. Radzikowskiego 152, 31-342 Kraków, Poland

27 Institut für Astro- und Teilchenphysik, Leopold-FranzensUniversität Innsbruck, 6020 Innsbruck, Austria

28 Obserwatorium Astronomiczne, Uniwersytet Jagielloński, ul. Orla 171, 30-244 Kraków, Poland

29 Toruń Centre for Astronomy, Nicolaus Copernicus University, ul. Gagarina 11, 87-100 Toruń, Poland

30 School of Chemistry \& Physics, University of Adelaide, Adelaide 5005, Australia

31 Charles University, Faculty of Mathematics and Physics, Institute of Particle and Nuclear Physics, V Holešovičkách 2, 18000 Prague 8, Czech Republic

32 School of Physics \& Astronomy, University of Leeds, Leeds LS2 9JT, UK

33 European Associated Laboratory for Gamma-Ray Astronomy, jointly supported by CNRS and MPG

34 Oskar Klein Centre, Department of Physics, Royal Institute of Technology (KTH), Albanova, 10691 Stockholm, Sweden 